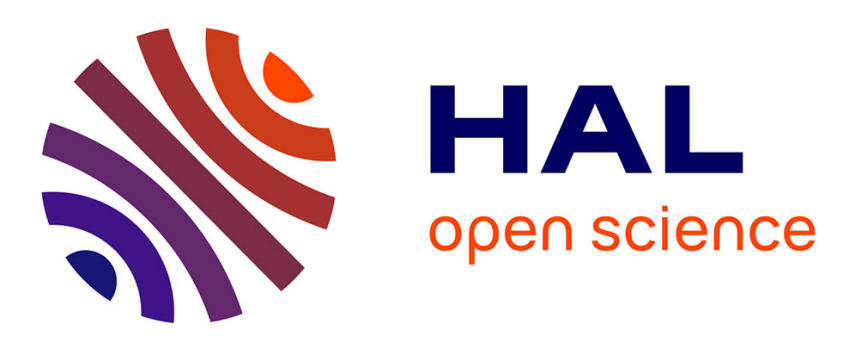

\title{
Démontrer la capacité de l'agriculture à relever le défi énergétique
}

\author{
Sophie Carton, Dominique Tristant, Yves Python, Thierry Doré, Thierry \\ Bonaudo
}

\section{- To cite this version:}

Sophie Carton, Dominique Tristant, Yves Python, Thierry Doré, Thierry Bonaudo. Démontrer la capacité de l'agriculture à relever le défi énergétique: L'agriculture face à de multiples défis. Pour : enquêtes et témoignages, 2013, 217, pp.37-45. hal-01173283

\section{HAL Id: hal-01173283 \\ https://hal.science/hal-01173283}

Submitted on 6 Aug 2019

HAL is a multi-disciplinary open access archive for the deposit and dissemination of scientific research documents, whether they are published or not. The documents may come from teaching and research institutions in France or abroad, or from public or private research centers.
L'archive ouverte pluridisciplinaire HAL, est destinée au dépôt et à la diffusion de documents scientifiques de niveau recherche, publiés ou non, émanant des établissements d'enseignement et de recherche français ou étrangers, des laboratoires publics ou privés. 


\section{DÉMONTRER LA CAPACITÉ DE L'AGRICULTURE À RELEVER LE DÉFI ÉNERGÉTIQUE}

Sophie Carton, Dominique Tristant, Yves Python, Thierry Doré et Thierry Bonaudo

GREP | « Pour »

2013/2 N²18 | pages 35 à 45

ISSN 0245-9442

Article disponible en ligne à l'adresse :

https://www.cairn.info/revue-pour-2013-2-page-35.htm

Distribution électronique Cairn.info pour GREP.

(C) GREP. Tous droits réservés pour tous pays.

La reproduction ou représentation de cet article, notamment par photocopie, n'est autorisée que dans les limites des conditions générales d'utilisation du site ou, le cas échéant, des conditions générales de la licence souscrite par votre établissement. Toute autre reproduction ou représentation, en tout ou partie, sous quelque forme et de quelque manière que ce soit, est interdite sauf accord préalable et écrit de l'éditeur, en dehors des cas prévus par la législation en vigueur en France. Il est précisé que son stockage dans une base de données est également interdit. 


\section{dossier 1 ère partie \\ Économies d'énergie et précarité énergétique}




\section{Sophie CARTON}

Ingénieur d'étude, ferme expérimentale d'AgroParisTech, programme Grignon Énergie positive

\section{Dominique TRISTANT}

Directeur de la ferme expérimentale d'AgroParisTech

\section{Yves PYTHON}

Ingénieur d'étude, ferme expérimentale d'AgroParisTech, programme Grignon Énergie positive

\section{Thierry DORÉ}

Professeur d'agronomie et directeur scientifique d'AgroParisTech

Maître de conférences, UMR SAD-APT, AgroParisTech

\section{Démontrer la capacité de l'agriculture à relever le défi énergétique}

\section{L'agriculture face à de multiples défis}

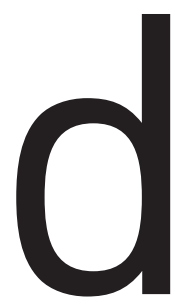

ans un contexte de volatilité accrue des prix agricoles et de concurrence croissante dans un marché mondialisé, la compétitivité des filières agroalimentaires est un

enjeu stratégique, tout particulièrement en France où l'agroalimentaire est un secteur exportateur net. En plus de ces contraintes 
économiques, l'agriculture et l'élevage sont de plus en plus confrontés à leurs impacts environnementaux : pollutions diffuses des sols et de l'eau Initrates, phosphates, pesticides), changement climatique (émissions de gaz à effet de serre), pertes de biodiversité (FAO, 2002).

Par ailleurs, l'agriculture et l'élevage sont dépendants des énergies fossiles Igaz, fioul, engrais azotés), ce qui constitue un enjeu à la fois pour l'environnement, pour la compétitivité des exploitations et pour la sécurité alimentaire des pays (Vert et Portet, 2010), dans un contexte où la production agricole mondiale doit augmenter pour répondre à une demande croissante. Ainsi, d'après la FAO (2011), pour nourrir une population mondiale estimée à 9,2 milliards d'individus en 2050, la production agricole mondiale devrait augmenter de $70 \%$ par rapport à la production moyenne 2005-2007. Selon les résultats du scénario tendanciel du projet Agrimonde, la consommation de calories finales pour l'alimentation humaine et animale et les autres usages devrait augmenter de $88 \%$ entre 2003 et 2050 (Paillard et al., 2009). Concilier la compétitivité, l'exigence de production et la préservation de l'environnement dans le domaine de l'agriculture est une équation difficile à résoudre. Pour trouver des solutions à tout ou partie de l'équation, les initiatives portées par diverses institutions ou associations foisonnent. Pour la France on peut citer l'agriculture biologique, l'agriculture raisonnée, l'agriculture écologiquement intensive, l'agroécologie, l'agriculture de conservation, l'agriculture durable etc. La FAO prône quant à elle « l'intensification durable de la production agricole ». Tous ces modèles ont pour point commun la nécessité d'une transformation du modèle agricole basé sur les intrants (notamment l'énergie fossile), vers des systèmes de production intensifs en savoir, efficients et adaptés à leur milieu (FAO, 2011).

Nous allons présenter un programme de recherche-action qui s'inscrit dans ce contexte : le programme Grignon Énergie positive (GE+), qui a pour base la ferme expérimentale d’AgroParisTech à Grignon. Nous présenterons ses spécificités et son originalité ainsi que quelques résultats obtenus depuis 2005 sur les consommations d'énergie fossile. Enfin, nous donnerons quelques perspectives de développement du programme.

\section{Objectifs et principes du programme Grignon Énergie positive}

Le projet GE+ a été lancé fin 2005, sous l'impulsion d'Olivier Lapierre, professeur à AgroParisTech et directeur du Céréopa'. L'objectif général du projet est

1 Le Céréopa (Centre d'étude et de recherche sur l'économie et l'organisation des productions animales) est un bureau d'études (association loi 1901) qui fonctionne au sein d'AgroParisTech (www.cereopa.com). 
de faire la démonstration que l'agriculture est capable de répondre aux enjeux environnementaux tout en restant rentable dans le contexte socio-économique actuel et en maintenant son niveau de production alimentaire, évalué à travers son potentiel nourricier.

Le potentiel nourricier d'une exploitation est un indicateur développé par le Céréopa qui désigne le nombre théorique de personnes qu'une ferme peut nourrir pendant un an. Il s'obtient en faisant le calcul suivant :

(Valeur nutritionnelle des matières premières vendues - Valeur nutritionnelle des matières premières achetées) Besoins moyens quotidiens d'un individu de référence (données issues de la FAO).

La valeur nutritionnelle des matières premières peut être exprimée selon trois critères : l'énergie (exprimée en calories), les protéines (animales + végétales) ou les protéines animales seules. Ce calcul ne prend en compte que les calories ou les protéines valorisables en alimentation humaine. Le devenir des matières premières, en aval de l'étape de production agricole, n'est pas pris en compte (Lapierre et Lapierre 2010).

Par le biais de la démonstration, le projet vise également à proposer aux exploitants agricoles un cadre méthodologique permettant de répondre aux défis économiques, environnementaux et sociétaux de l'agriculture, traités de façon simultanée et symbolisés dans la figure 1 (Carton et al. 2009, Lapierre et al. 2010).

Figure 1. Triangle des performances du projet GE+

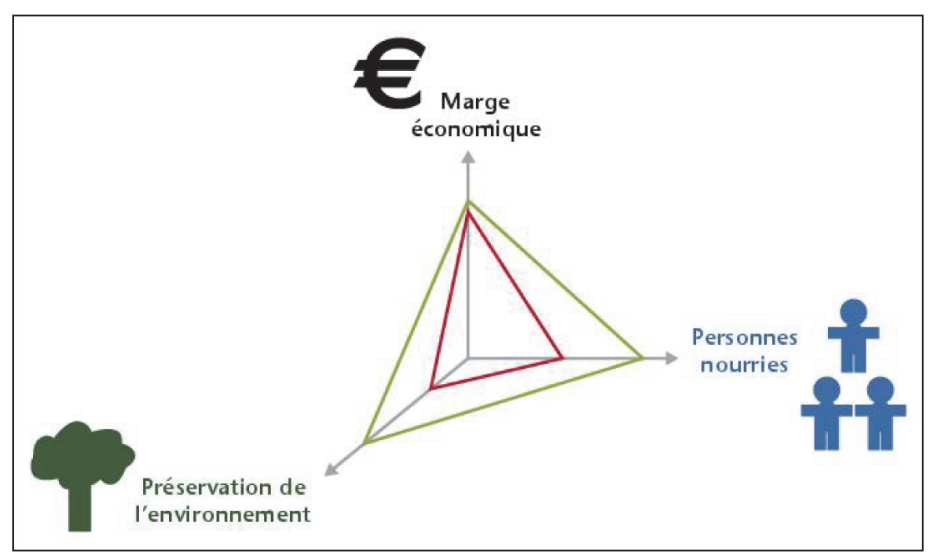

Pour cela, le projet comprend un axe de travail technique mais aussi un volet de valorisation des résultats vers les professionnels, les institutionnels et le grand public.

Sur le plan technique, la démonstration menée à la ferme de Grignon se fonde sur une démarche de progrès focalisée sur 
la diminution des consommations d'énergie fossile et des émissions de gaz à effet de serre des activités agricoles.

Cette démarche de progrès a été mise au point à la ferme de Grignon entre 2006 et 2009 (Acte 1 du projet). Elle consiste en un processus d'amélioration continue décomposé en cinq étapes (Bonaudo et al. 2010, Lapierre et al. 2010) :

1. l'inventaire des solutions techniques disponibles;

2. la sélection des actions potentiellement les plus efficaces et les mieux adaptées au système à l'aide d'un logiciel d'optimisation du fonctionnement de l'exploitation agricole : PerfAgroP ${ }^{3} \circledast$. Ce logiciel est utilisé pour tester l'effet de changements de pratiques de culture ou d'élevage sur le résultat économique, les performances productives, les émissions de GES et l'énergie consommée (évaluation ex-ante) ;

3. la définition d'un plan d'action à l'échelle de l'exploitation ;

4. la mise en pratique des voies de progrès ;

5. Le suivi dans le temps, par des mesures de flux de matière et d'énergie, de l'évolution des consommations d'énergie et des émissions de gaz à effet de serre de l'exploitation (évaluation ex-post).

À la différence d'autres initiatives traitant de durabilité en agriculture, la démarche de progrès Grignon Énergie positive ne se fonde pas sur la proposition d'une ou de plusieurs bonnes pratiques ou d'un modèle de production en particulier lobjectifs de moyens) comme en agriculture de conservation ou en agriculture biologique. Elle repose sur des objectifs de minimisation des consommations d'énergie fossile et des émissions de gaz à effet de serre tenant compte des contraintes de l'exploitation (objectifs de résultats), qu'elles soient d'ordre économique, politique (système de subvention), social, spatial, technique, etc. Grignon Énergie positive propose donc une méthode et des outils permettant de tester puis de mettre en place des solutions techniques ou organisationnelles les plus efficaces. Les solutions techniques trouvées peuvent tout à fait relever des différents modèles ou démarches cités précédemment.

\section{La transformation à l'œuvre à la ferme de Grignon}

En 2006, la ferme comptait 517 hectares de surface agricole utile, décomposés comme suit : 187 ha de blé tendre, 90 ha de maïs, 77 ha d'orge d'hiver, 50 ha d'orge de printemps, 6 ha de colza, 6 ha de féverole, 73 ha de prairies permanentes et 28 ha de prairies temporaires.

La ferme comptait également un élevage de 420 brebis-mères et leur suite (120 agnelles de renouvellement, 720 agneaux de vente), ainsi qu'un élevage de 110 vaches laitières avec une productivité annuelle moyenne de 9200 litres de lait standard par vache, 90 génisses de renouvellement et 58 veaux vendus. 
Une veille bibliographique scientifique et technique faite tout au long de l'année 2006 a servi à inventorier l'ensemble des leviers d'actions permettant de découpler l'évolution de la production agricole de celle des consommations d'énergie et des émissions de gaz à effet de serre.

Les conséquences de l'adoption des bonnes pratiques les plus applicables au cas de la ferme de Grignon len termes techniques, économiques, réglementaires, etc.), ont ensuite été testées avec l'outil PerfAgroP3® pour évaluer leur impact potentiel sur la marge économique, les consommations d'énergie fossile, les émissions de gaz à effet de serre et le potentiel nourricier.

L'évaluation ex-ante a été répétée plusieurs fois depuis 2006, au fur et à mesure de l'évolution du contexte de l'exploitation, et ce, dans une optique d'amélioration continue. Elle a permis de sélectionner les solutions suivantes, qui ont été mises en œuvre progressivement à partir de 2007 :

- l'augmentation de la production de lait 19200 litres de lait standard par vache par an en 2006 à 10400 litres de lait standard par vache par an en 2012) ;

- la diminution du coût énergétique des aliments autoproduits avec l'insertion de luzerne autoproduite dans les rations à partir de 2007 (foin et ensilage) et la réintroduction du pâturage (pour les génisses et les vaches taries à partir de 2007. pour une partie des vaches en lactation à partir de 2010) ;

- la diminution du coût énergétique des aliments achetés avec le remplacement du tourteau industriel par du tourteau de colza gras (produit localement avec une presse semi-industrielle à partir du colza fourni par la ferme d'AgroParisTech, qui est $20 \%$ moins consommateur d'énergie que la référence Diaterre (Ademe 2011)l et l'arrêt du recours aux produits déshydratés ;

- la diversification des cultures, avec l'introduction de la luzerne à partir de 2007 (30 ha en 2012), du triticale à partir de 2010 (22 ha en 2012), l'augmentation de la surface en colza (36 ha en 2012) et en prairies temporaires ( 9 ha supplémentaires en 2010), associée à une diminution des surfaces en blé et en maïs (réduites respectivement à 97 ha et 55 ha en 2012) ;

- la meilleure valorisation du potentiel fertilisant des effluents (l'azote organique est passé de $31 \%$ de l'azote total (azote épandu + azote symbiotique) en 2006 à $42 \%$ en 2012) et le renforcement du pilotage de la fertilisation (utilisation d'outils d'aide à la décision et analyses de soll ;

- l'application de techniques culturales simplifiées associée au ramassage de la menue paille (à partir de 2008) ;

- l'augmentation de la longévité des vaches pour diminuer le nombre de génisses de renouvellement lle ratio du nombre de génisses sur le nombre de vaches en lactation est passé de 0,85 en 2006 à 0,62 en 2012). 
Des événements sans lien avec la démarche d'amélioration ont également modifié le système de la ferme de Grignon :

- une augmentation de quota de 100000 litres de lait en 2012, l'effectif de l'élevage a donc été porté à 135 vaches laitières;

- la perte de 94 hectares de surface cultivée au total en 2011 et 2012.

\section{Des résultats contrastés en matière de consommation d'énergie}

Les évolutions des performances globales de l'exploitation présentées dans ce paragraphe ne sont pas exprimées en valeur brute mais en valeur relative au potentiel nourricier de l'exploitation. En effet, l'enjeu de la démarche de progrès $\mathrm{GE}+$ est de diminuer au maximum les consommations d'énergie et les émissions de gaz à effet de serre de la production agricole sans remettre en cause sa vocation première qui reste de nourrir les humains.

Une réduction de $10 \%$ des consommations d'énergie par personne potentiellement nourrie selon les besoins en protéines a été mesurée à la ferme de Grignon entre 2006 et 2012. Cependant, la progression a été très aléatoire puisque les consommations d'énergie par personne nourrie de l'année 2011 ont été supérieures à toutes les autres années prises en compte (2006 à 2012) (Figure 2).

Ce phénomène s'explique d'abord par la perte de surfaces en cultures de vente à partir de 2011, qui a davantage réduit le potentiel nourricier de l'exploitation que ses consommations d'énergie. En effet, alors que les cultures de vente de la ferme de Grignon représentent entre 55 et $80 \%$ du potentiel nourricier de l'exploitation exprimé en protéines totales selon les années, elles ne sont à l'origine que de 20 à $40 \%$ des consommations d'énergies totales. Cela explique aussi qu'en 2012, la ferme de Grignon ne retrouve pas son niveau de performance de 2010.

La situation de l'année 2011 s'explique ensuite par des conditions climatiques très défavorables aux cultures d'hiver, qui ont provoqué une sous-production de 16,1 tonnes de protéines végétales, soit 840 personnes potentiellement nourries en moins. 
Figure 2. Consommations d'énergie fossile par personne nourrie à la ferme de Grignon (2006-2012)

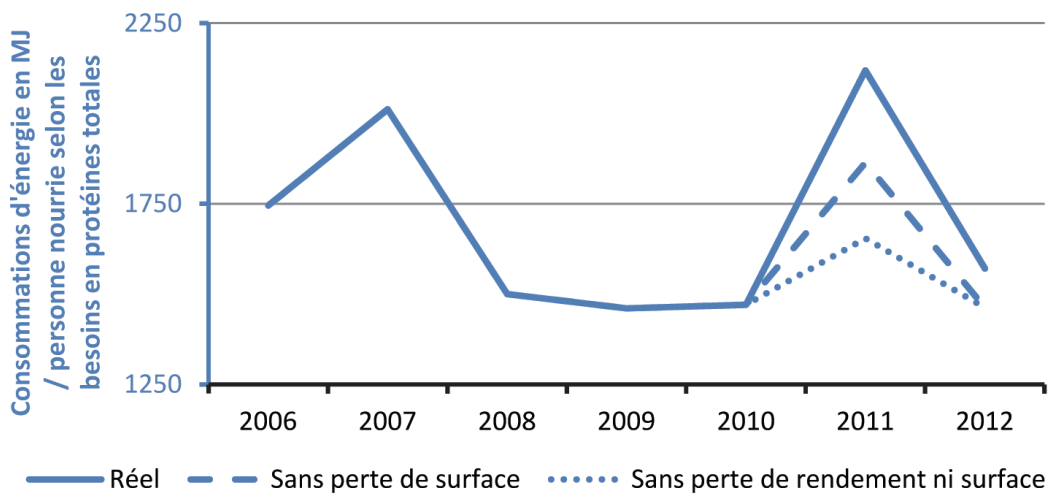

Concernant l'atelier laitier, les consommations d'énergie fossile ont diminué de $34 \%$ environ entre 2006 et 2012. Les gains obtenus sont relativement stables (Figure 3).

Figure 3. Consommations d'énergie fossile de l'atelier laitier à la ferme de Grignon (2006-2012) avec allocation économique entre lait et viande

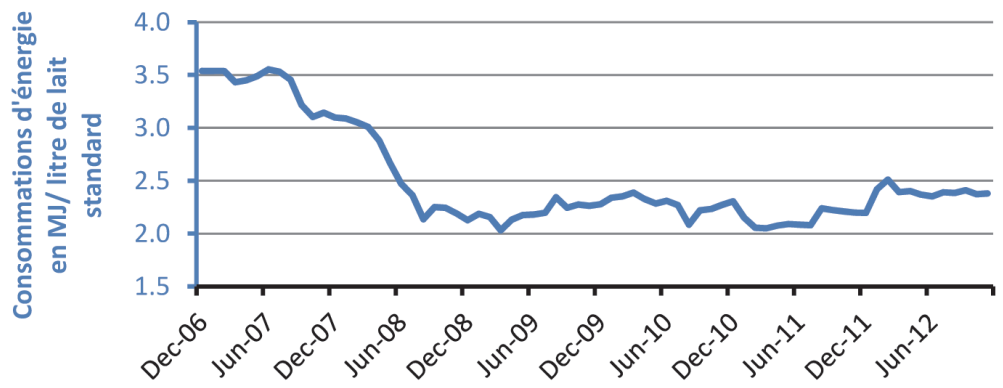

Environ $90 \%$ de la diminution des consommations d'énergie par litre de lait s'expliquent par l'alimentation des vaches. À titre d'illustration, la quantité de matière sèche d'aliments ingérés par litre de lait standard produit a diminué d'environ $13 \%$ entre 2006 et 2011 grâce à l'augmentation de la productivité des animaux, et les consommations d'énergie fossile par unité de matière sèche d'aliments ingérés ont diminué d'environ 33 \% sur la même période. 


\section{Conclusion et projets}

La démarche de progrès GE+ continue d'être appliquée à la ferme de Grignon. Elle a permis de trouver des solutions pour améliorer les pratiques de culture et d'élevage de l'exploitation et a généré en particulier une diminution significative des consommations d'énergie fossile de l'atelier laitier. La ferme de Grignon a ainsi atteint un bon niveau de performance énergétique, comme peuvent en attester les comparaisons avec des références nationales sur la production laitière de l'Institut de l'élevage (2011) (2,1 à 5,3 MJ/litre de lait standard selon les types de système, ces valeurs prenant en compte cinq postes de consommation qui représentent environ $90 \%$ des dépenses en énergie des élevages herbivores). L'optimisation se poursuit, avec par exemple en 2012 l'investissement dans un refroidisseur à plaques installé en sortie de salle de traite. Cependant, la stagnation des performances depuis 2008 semble montrer qu'il est difficile d'aller plus loin dans la réduction des consommations d'énergie fossile, en tout cas avec les solutions employées jusqu'à maintenant. Ainsi, une réflexion sur l'installation d'une unité de méthanisation pour produire de l'électricité et de la chaleur est en cours.

Un intérêt majeur de la démarche est de fournir un cadre méthodologique dans lequel un grand nombre de pratiques différentes ou de modèles de production peuvent s'insérer. Ainsi, la démarche est aujourd'hui déployée dans un réseau de 25 fermes réparties sur le territoire français, recouvrant une grande variété de systèmes et de stratégies de production.

Enfin, sept années de suivi de la performance à la ferme de Grignon ont permis de mettre en évidence une limite importante de la démarche de progrès, qui est la difficulté de traiter le risque encouru par les exploitations agricoles. Les années 2011 et 2012 ont en effet montré que, malgré les bonnes pratiques mises en œuvre, l'exploitation reste soumise à un fort aléa qui joue sur ses résultats technique et économique, mais aussi sur son impact environnemental. Un enjeu fort pour les années à venir est la meilleure prise en compte de ce risque, en particulier dans l'évaluation ex-ante, afin de trouver des solutions pour augmenter la robustesse des exploitations et les rendre performantes même les mauvaises années.

\section{À lire}

Thierry Bonaudo, Sophie Carton, Dominique Tristant et Olivier Lapierre, Cadre d'action pour l'émergence d'une agriculture écologiquement intensive : le programme Grignon Énergie positive, 2010. 
Olivier Lapierre, Sophie Carton, Dominique Tristant, Yves Python, Bernard de Franssu, Aline Lapierre et Marion Barral, «Le projet pilote de ferme à bilan énergétique positif et les complémentarités productions animales et végétales », OCL, vol. 17, n 5, septembre-octobre 2010. Sophie Carton, Marion Barral et Olivier Lapierre, Grignon Énergie positive... vers l'Acte II, 2009. FAO, Agriculture mondiale : horizon 2015/2013, Rapport abrégé, 2002.

FAO, Save and Grow - A Policymaker's guide to the sustainable intensification of smallholder crop production, 2011.

Aline Lapierre et Olivier Lapierre, Le calculateur de performance nourricière PerfAlim -Guide méthodologique, 2010.

Julien Vert et Fabienne Portet, (coord.), «Prospective Agriculture Énergie 2030. L'agriculture face aux défis énergétiques », Centre d'études et de prospective, SSP, Ministère de l'Agriculture, de l'Alimentation, de la Pêche, de la Ruralité et de l'Aménagement du territoire, 2010.

Ademe, «Guide des valeurs Dia'terre ${ }^{\circledR}$ », version du référentiel 1.11, 2011.

Sandrine Paillard, Sébastien Treyer et Bruno Dorin, (coord.), Agrimonde. Scénarios et défis pour nourrir le monde en 2050, éd. Quae, coll. « Matière à débattre et décider », 2010.

Institut de l'élevage, (éd.), Consommations d'énergie en élevages herbivores et leviers d'action, Coll. « Méthodes et Outils », 2011.

Grignon Énergie positive. Un pôle de référence sur la thématique agriculture, énergie et changement climatique. Disponible sur : http://www.grignonenergiepositive.fr 